\title{
Colorectal surgical outcomes following implementation of an enhanced recovery after surgery programme in Cape Town
}

\author{
R Oodit, ${ }^{1,2,3}$ (iD DA Constant, ${ }^{4}$ (D) F Maree, ${ }^{5}$ (D) I Lorrimer, ${ }^{5}$ (D) EK Dalwai, ${ }^{1,6}$ (D) J Moodley ${ }^{4,7}$ (iD) \\ ${ }^{1}$ Department of Surgery, University of Cape Town, South Africa \\ ${ }^{2}$ Department of Global Surgery, University of Cape Town, South Africa \\ ${ }^{3}$ Dr Matley and Partners, Constantiaberg MediClinic Hospital, South Africa \\ ${ }^{4}$ Women's Health Research Unit, School of Public Health and Family Medicine, Faculty of Health Sciences, University of Cape Town, \\ South Africa \\ ${ }^{5}$ Dr Matley and Partners, Vincent Palotti Life Health Hospital, South Africa \\ ${ }^{6}$ Dr Matley and Partners, Wilderness Road, Claremont, South Africa \\ ${ }^{7}$ Cancer Research Initiative, Faculty of Health Sciences, University of Cape Town, South Africa
}

Corresponding author, email: ravioodit@gmail.com

Background: Colorectal surgery is commonly performed for benign and malignant colorectal disease. The aim of this study was to describe length of stay (LOS), complications and its associated factors in patients undergoing elective colorectal surgery following implementation of an enhanced recovery after surgery (ERAS) programme in South Africa (SA).

Methods: Socio-demographic, pre- intra- and postoperative clinical details and compliance to the ERAS guidelines were recorded in all patients undergoing colorectal surgery in a private practice in Cape Town, SA. Means and standard deviations or medians and interquartile range (IQR), as appropriate, were used to describe continuous variables and frequencies and percentages for categorical variables. Bivariate and multivariate analyses using linear regression of $\log$ transformed LOS and logistic regression for development of complications were performed.

Results: Between 2015 and 2019, 457 patients had elective colorectal surgical procedures. The median LOS was 5 days (IQR 3-7). Pre- and intraoperative compliance was 92\% and 86\% respectively. In total, 203 (44\%) patients developed 346 complications, of which $61 \%$ were minor. On bivariate analysis, increased intraoperative compliance was associated with a significant decrease in LOS (coefficient $[\beta]=0.987,95 \%$ confidence interval [CI] $0.984-0.991$ ) and complications (odds ratio [OR] $0.457,95 / 5 \mathrm{CI} 0.266-0.787$ ). For every additional 30 minutes of theatre time, irrespective of type of procedure, LOS increased by $8 \%$ and complications by $12 \%$. On multivariate analysis, laparoscopic compared to open surgery was also associated with a shorter $\operatorname{LOS}(\exp [\beta]=0.667,95 \%$ CI $0.580-0.767 p<0.001)$ and reduced complications (OR $0.457,95 \%$ CI $0.266-0.787$ ).

Conclusion: Our results show that high compliance to the ERAS guidelines is possible in the private sector in SA and that a significant reduction in LOS can be achieved without placing the patient at a higher risk of complications.

Keywords: colorectal surgery, perioperative care, ERAS, LMIC, surgical checklist

\section{Introduction}

Colorectal surgery is commonly performed for both benign and malignant colorectal disease. Length of stay (LOS) and complication rates are commonly used as surrogate markers of quality and efficiency of perioperative care. ${ }^{1}$ Data from high-income countries (HICs) show that patients undergoing colorectal surgery under traditional care typically have a LOS of 10-14 days and that $15-48 \%$ develop complications. ${ }^{2}$ In the last 30 years, there have been significant changes in perioperative care, targeted at reducing the severe catabolic stress response to surgery and improving insulin sensitivity., The enhanced recovery after surgery (ERAS) programme was established in 2010 to improve perioperative care through implementation of these and other findings. The programme includes a set of evidence-based perioperative management guidelines, an implementation programme and a monitoring and evaluation system. ${ }^{5}$ Randomised controlled trials conducted in HICs have shown that for colorectal surgery the ERAS programme reduced LOS to 5-7 days (20-40\%) and complication rates by $20-30 \% .6{ }^{6,7}$ Implementation of ERAS programmes has also reduced in-hospital costs by $10-20 \%{ }^{8,9}$ The Alberta Study demonstrated a return on investment of $\$ 3.8: 1$ (i.e., a saving of $\$ 3.8$ for every $\$ 1$ invested in the programme). ${ }^{10}$

There is limited LOS and complication data for colorectal surgery from low- and middle-income countries (LMICs), including South Africa (SA). ${ }^{11-14}$ We introduced an ERAS programme for colorectal surgery into a private surgical group practice in Cape Town, SA, in April 2015. In this paper we report on an audit of the implementation of this ERAS programme and its outcomes. Compliance to the ERAS 
guidelines, LOS, complications and associated factors for patients who had elective colorectal surgery are described.

\section{Method}

\section{Implementation of the ERAS programme in Cape Town}

The ERAS programme included establishing a dedicated perioperative ERAS multidisciplinary team (MDT), implementation of the ERAS guidelines and setting up an ERAS database. The MDT included two ERAS-trained nurse coordinators, nine surgeons of whom, two had a colorectal sub-specialty qualification, five anaesthetists, three physiotherapy teams, and two dieticians. The ERAS Society's 22 evidence-based colorectal guidelines were reviewed by the MDT and implemented without any change. ${ }^{5}$ The lead surgeon and ERAS nurse coordinators met weekly and the entire team quarterly as part of the PlanDo-Study-Act (PDSA) cycle that was adopted. ${ }^{15}$

\section{Recruitment and data entry}

Verbal consent to collect clinical data was obtained from all patients 18 years and older undergoing elective colorectal and small bowel surgery. Data was entered into the ERAS database by the nurse coordinator and all data was anonymised.

\section{Measurements}

We measured socio-demographic (age and gender), pre-, intra- and postoperative clinical details and compliance to the ERAS guidelines.

\section{Preoperative variables}

Wemeasured weight(in kilograms)and height(in centimetres) and calculated a body mass index (BMI). Patients with a BMI $\geq 30$ were classified as obese. The American Society of Anesthesiologists (ASA grade 1-4) and the physiological operative score for enumeration of mortality and morbidity (POSSUM) risk scores for complications were recorded. The ASA grade was categorised into low risk (grade 1\&2) or high risk (grade $3 \& 4$ ). The malnutrition universal screening tool (MUST) was used to identify patients who were either malnourished or at risk of malnourishment. Patients who had stopped smoking for more than 30 days were deemed non-smokers. Additional measures recorded were: HbAlc for diabetics, diagnosis on presentation, radiotherapy treatment received preoperatively, the use of a $12.5 \%$ complex carbohydrate drink up to 2 hours prior to surgery and whether the patient received preoperative counselling with the ERAS nurse.

\section{Intraoperative variables}

Surgical procedures were grouped into either colectomy or rectal resections. For each, the procedure was laparoscopic, laparoscopic converted to open or planned open. The duration of the surgical procedure reflects the total of surgical and anaesthetic time recorded in minutes and then aggregated into 30-minute increments. The type of anaesthesia was categorised as total intravenous anaesthesia (TIVA) or inhalational. The use of an epidural was defined as compliant for open procedures and non-compliant for laparoscopic procedures. The following were also documented: the administration and timing on antibiotic prophylaxis, the use of a warming blanket, patient temperature on completion of the procedure, the quantity of intravenous fluid administered and the use of long and short acting intravenous opioids.

\section{Postoperative variables}

The following were recorded daily: quantity (litres) and duration (days) of intravenous fluids, time to passage of flatus and stools, the tolerance of oral fluids and a normal diet, and the use of long and short acting opioids in the first 48 hours. Day 0 (D0) for intravenous fluids was defined as total fluids administered on the day of surgery until $08 \mathrm{~h} 00$ the following day. Compliance to D0 fluids is defined as $3 \mathrm{~L}$ for colectomy and 3.5 L for rectal surgery.

LOS was defined as the duration (nights) spent in hospital from the date of admission to discharge. Any patient requiring readmission and or repeat surgery within 30 days after the index operation was recorded.

All complications occurring within 30 days of the procedure were recorded. The complication rate was calculated as the total number of complications divided by the total number of patients undergoing surgery. The Clavien-Dindo grade classification was used for complication events as defined in the ERAS guidelines. ${ }^{16}$

All data for each patient were entered by the ERAS nurse onto the ERAS database. The calculation of compliance (yes/no) to the ERAS guidelines was generated by the ERAS software. Any missing compliance data were recorded as non-compliant. Overall compliance reflects the average of the pre-, intra- and postoperative compliance.

The main outcome measures were LOS and complications. The impact of the degree of compliance to the preand intraoperative guidelines on LOS for colon and rectal procedures and on complications was assessed.

\section{Data analysis}

Data analysis was conducted using STATA v.15 (StataCorp LLC, College Station, TX). For continuous variables, we used means and standard deviations for normally distributed variables and interquartile range where distributions were significantly skewed (Shapiro-Wilk test, $p<0.05$ ). For categorical variables we used frequencies and percentages. We assessed the association between the main dependent variables and the independent variables of age, gender, BMI, ASA and P-POSSUM score, smoking, diabetes, previous radiotherapy, preoperative counselling, the use of preoperative carbohydrate drinks, the type of procedure and anaesthesia, surgical approach, duration of the procedure, hypothermia, postoperative opioid administration, compliance to the preoperative and intraoperative compliance guidelines.

We performed bivariate and multivariate analyses using linear regression of $\log$ transformed LOS and logistic regression for development of complications. Variables included in the regression analyses were selected a priori according to evidence and clinical judgment and included patient profile, characteristics and outcomes of surgery, and pre- and intraoperative compliance measures. R2 statistic and $t$ values of each beta coefficient were calculated. Statistical significance levels were set at $p<0.05$ (Supplementary Table S1). 


\section{Results}

\section{Patient profile}

Between April 2015 and November 2019, a total of 481 patients were eligible for enrolment into the ERAS programme. One patient declined. Of the remaining 480 patients, 457 had colorectal and 23 had small bowel surgical procedures. In this study, we report on the 457 patients who had elective colorectal surgical procedures. Of these patients, 290 had a colonic resection and 167 a rectal resection. The median age was 65 years (IQR 56-73 years), with $25 \%$ of patients 73 years and older. The key demographic and clinical data are summarised in Table I.

The most common indication for surgery was a diagnosis of cancer - 322 patients (70\%). Of the 137 patients with benign disease, 47 patients (34\%) had benign polyps, and $44(32 \%)$ diverticular disease. In terms of surgical procedures, $69 \%$ had a laparoscopic procedure, $31 \%$ an open procedure and $5 \%$ were converted from laparoscopic to an open procedure. Median theatre time for open surgery was significantly shorter at 2 hours and 15 minutes (IQR $1 \mathrm{~h} 38 \mathrm{~m}-2 \mathrm{~h} 02 \mathrm{~m}$ ) than laparoscopic surgery, 3h $13 \mathrm{~m}$ ([IQR $2 \mathrm{~h} 27 \mathrm{~m}-3 \mathrm{~h} 47 \mathrm{~m}], p<0.001)$. Bivariate logistic regression showed that for every 30-minute increase in theatre time, irrespective of type of procedure, the likelihood of developing a complication increased by $12 \%(\mathrm{OR}=1.12, p$ $=005,95 \%$ CI $1.03-1.22)$ and LOS increased by $8 \%$ (OR $=1.08, p=0.000[p<0.001] 95 \%$ CI 1.06-1.11).

Most patients $(78 \%)$ received the appropriate volume of intravenous fluids on Day 0 , while $2 \%$ received $>5 \mathrm{~L}$. The majority of patients $(98 \%)$ were able to tolerate more than $1 \mathrm{~L}$ of fluids orally on day 1 and $85 \%$ had a full ward diet by Day 3. By Day 1 post-surgery $95 \%$ of the patients had passed flatus. Fifty-one per cent of patients passed a stool by Day 3 .

\section{Compliance to the ERAS guidelines}

The pre-, intra- and postoperative compliance was $94 \%$, $86 \%$ and $64 \%$ respectively over the 5 -year study period. Figure 1 depicts compliance per year. Pre- and intraoperative

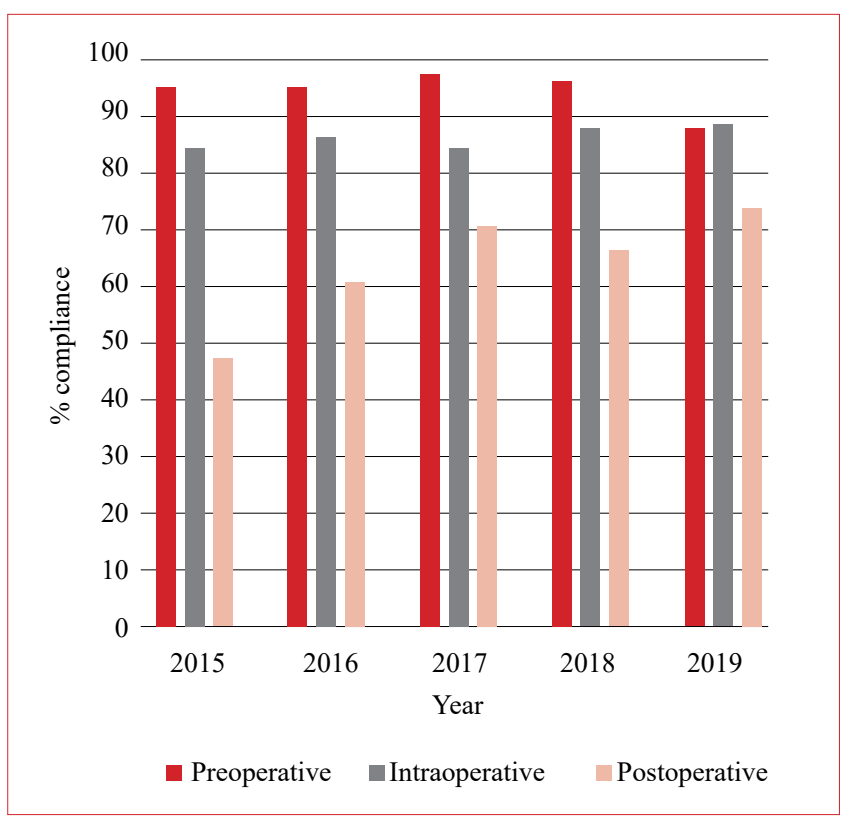

Figure 1: Compliance per year
Table I: Demographic and clinical data

$n(\%)$

$\begin{array}{ll}\text { Gender } & \\ \text { Male } & 226(49.5)\end{array}$

Female

$231(50.5)$

BMI $\left(\mathrm{kg} / \mathrm{m}^{2}\right)$

$<30$

$333(73.4)$

$\geq 30$

\section{Diabetes}

No

$388(84.9)$

Yes

Smoker

No

Yes

ASA class

$1-2$

3-4

P-POSSUM score administered

No

$46(10.1)$

Yes

MUST score

1

$400(90.5)$

$>1$

Previous radiotherapy - rectal cancer

No

$113(67.6)$

Yes

Preoperative carbohydrate

No

Yes

Preoperative counselling

No

Yes

Indication for surgery

Cancer

Benign disease

Procedure type

Rectal resection

$167(36.5)$

Colectomy

Surgical approach

Open

$119(26.0)$

Laparoscopic converted to open

Laparoscopic

$316(69.2)$

Anaesthetic

Volatile

TIVA

$279(61.3)$ 
compliance was above $80 \%$ throughout the study period. Postoperative compliance was low at commencement (47\%) and improved to $74 \%$ in year 5 . Intraoperative compliance was significantly higher for those undergoing laparoscopic and laparoscopic conversion to open procedures compared to open procedures $(100 \%$ and $83 \%$ vs $80 \% ; p<0.001)$. The difference was due to the selective use of epidurals in open procedures. ERAS compliance had an inversely proportional effect on length of stay.

\section{Length of stay}

The overall median LOS was 5 days (IQR 3-7). The LOS for both laparoscopic and open colectomy procedures was 4 days (IQR 3-7), laparoscopic rectal procedures was 5 days (IQR 4-9) and open rectal procedures was 7 days (IQR 5-10). The overall readmission rate was $12 \%$ (55).

On bivariate analysis, several factors were associated with LOS (Table II). On regression analysis (Table III), factors associated with a significantly shorter LOS were: laparoscopic surgery compared to open surgery (exp [B] $=$ $0.667,95 \%$ CI $0.580-0.767, p<0.001)$ and appropriate antibiotic prophylaxis $(\exp [\beta]=0.598,95 \%$ CI $0.383-0.933$, $p=0.023)$. Factors associated with a longer LOS were: age $(\exp [\beta]=1.005,95 \%$ CI $1.001-1.010, p=0.010)$; theatre time $(\exp [\beta]=1.003,95 \%$ CI 1.002-1.004, $p<0001)$; postoperative hypothermia $(\exp [\beta]=1.199,95 \%$ CI 1.044 $1.378, p=0.01)$ and an antomotic leak $(\exp [\beta]=1.851$, CI $1.448-2.304, p<0.001)$.

\section{Complications}

A total of 203 (44\%) patients developed 346 complications, the majority $(61 \%)$ were minor. The complications and the Clavien-Dindo grade for complication events is shown in Table IV. Factors associated with complications on bivariate analysis are presented in Table II. On regression analysis, laparoscopic compared to open surgery was associated with fewer complications (OR 0.457, 95\% CI 0.266-0.787, $p=0.005)$. The following were associated with increased complications: smoking (OR 1.846, CI 1.031-3.304, $p=0.039)$ and theatre time (OR 1.006, 95\% CI 1.003 $1.009, p=0.001)$. Diabetes, obesity, older age, ASA score and rectal surgery were not associated with complications on regression analysis.

\begin{tabular}{|c|c|c|c|c|c|c|}
\hline \multirow[b]{2}{*}{ Risk factor } & \multicolumn{3}{|c|}{ Length of stay (days) } & \multicolumn{3}{|c|}{ Complication } \\
\hline & $\exp (ß)=$ & $95 \% \mathrm{CI}$ & $p$-value & OR & $95 \% \mathrm{CI}$ & $p$-value \\
\hline Age (years) & 1.005 & $1.001-1.010$ & 0.013 & 0.988 & $0.975-1.002$ & 0.098 \\
\hline Female & 0.957 & $0.852-1.075$ & 0.458 & 0.767 & $0529-1.112$ & 0.162 \\
\hline $\mathrm{BMI} \geq 30\left(\mathrm{~kg} / \mathrm{m}^{2}\right)$ & 1.061 & $0.931-1.210$ & 0.374 & 1.443 & $0.949-2.195$ & 0.087 \\
\hline $\mathrm{ASA}>2$ & 1.274 & $1.083-1.499$ & 0.004 & 1.107 & $0.660-1.857$ & 0.699 \\
\hline POSSUM & 1.007 & $0.832-1.220$ & 0.939 & 1.442 & $0.768-2.707$ & 0.254 \\
\hline Smoker & 1.065 & $0.905-1.252$ & 0.447 & 1.949 & $1.154-3.290$ & 0.013 \\
\hline Diabetic & 1.903 & $0.928-1.288$ & 0.286 & 1.317 & $0.788-2.201$ & 0.293 \\
\hline Preoperative radiotherapy & 1.412 & $1.183-1.686$ & $<0.001$ & 1.626 & $0.917-2.881$ & 0.096 \\
\hline Preoperative oral carbohydrate given & 1.020 & $0.823-1.263$ & 0.857 & 0.673 & $0.343-1.323$ & 0.251 \\
\hline Pre-admission counselling & 1.550 & $1.001-2.400$ & 0.050 & 2.481 & $0.495-12.429$ & 0.269 \\
\hline Preoperative compliance $(\%)$ & 0.999 & $0.999-1.005$ & 0.743 & 1.013 & $0.992-1.103$ & 0.228 \\
\hline \multicolumn{7}{|l|}{ Surgical approach } \\
\hline Open & Referent & - & - & Referent & - & - \\
\hline Laparoscopic & 0.739 & $0.648-0.843$ & $<0.001$ & 0.544 & $0.355-0.833$ & 0.005 \\
\hline Conversion to open & 0.909 & $0.687-1.203$ & 0505 & 0.883 & $0.349-2.238$ & 0.794 \\
\hline Surgery for rectal cancer & 1.229 & $1.091-1.384$ & 0.001 & 1.186 & $0.808-1.742$ & 0.383 \\
\hline Duration of procedure (minutes) & 1.003 & $1.002-1.003$ & $<0.001$ & 1.004 & $1.001-1.006$ & 0.005 \\
\hline Total intravenous general anaesthesia & 1.011 & $0.897-1.140$ & 0.852 & 0.967 & $0.660-1.416$ & 0.862 \\
\hline Antibiotic prophylaxis & 0.599 & $0.362-0.991$ & 0.046 & 0.813 & $0.162-4.072$ & 0.801 \\
\hline $\begin{array}{l}\text { Compliance with ERAS guideline for } \\
\text { normothermia intraoperatively }\end{array}$ & 0.819 & $0.512-1.310$ & 0.404 & 1.093 & $0.242-4.940$ & 0.908 \\
\hline Hypothermia (<36 Celsius) & 1.131 & $0.969-1.320$ & 0.118 & 0.817 & $0.495-1.348$ & 0.429 \\
\hline $\begin{array}{l}\text { Compliance with avoidance of opioids } \\
\text { intraoperatively }\end{array}$ & 0.952 & $0.838-1.080$ & 0.444 & 0.876 & $0.585-1.312$ & 0.520 \\
\hline Intraoperative compliance (\%) & 0.987 & $0.984-0.991$ & $<0.001$ & 0.984 & $0.972-0.996$ & 0.008 \\
\hline $\begin{array}{l}\text { Compliance with ERAS guideline for IV } \\
\text { fluid Day } 0\end{array}$ & 1.047 & $0.896-1.223$ & 0.563 & 1.342 & $0.813-2.214$ & 0.250 \\
\hline $\begin{array}{l}\text { Long-acting opioids given } 1 \text { st } 48 \text { hours } \\
\text { postoperatively }\end{array}$ & 1.425 & $1.165-1.743$ & $<0.001$ & 1.917 & $1.004-3.658$ & 0.049 \\
\hline Anastomotic leak & 2.035 & $1.608-2.674$ & $<0.001$ & N/A & N/A & N/A \\
\hline
\end{tabular}


Table III: Multivariable logistic regression of risk factors for length of stay (days) and logistic regression of risk factors for development of any complication

\begin{tabular}{|c|c|c|c|c|c|c|}
\hline \multirow[b]{2}{*}{ Risk factor } & \multicolumn{3}{|c|}{ Length of stay (days) } & \multicolumn{3}{|c|}{ Complication } \\
\hline & $\exp (B)=$ & $95 \% \mathrm{CI}$ & $p$-value & OR & $95 \% \mathrm{CI}$ & $p$-value \\
\hline Age (years) & 1.005 & $1.001-1.010$ & 0.010 & 0.992 & $0.976-1.008$ & 0.315 \\
\hline Female & 1.025 & $0.923-1.137$ & 0.648 & 0.759 & $0.507-1.136$ & 0.180 \\
\hline BMI $30 \geq\left(\mathrm{kg} / \mathrm{m}^{2}\right)$ & 1.042 & $0.922-1.178$ & 0.508 & 1.288 & $0.805-2.061$ & 0.291 \\
\hline ASA $>2$ & 1.128 & $0.964-1.321$ & 0.134 & 0.974 & $0.529-1.793$ & 0.932 \\
\hline P-POSSUM & 1.000 & $0.834-1.200$ & 0.999 & 1.281 & $0.622-2.628$ & 0.502 \\
\hline Smoker & 1.032 & $0.890-1.197$ & 0.677 & 1.846 & $1.031-3.304$ & 0.039 \\
\hline Diabetic & 0.995 & $0.851-1.164$ & 0.954 & 1.445 & $0.802-2.603$ & 0.220 \\
\hline Preoperative radiotherapy & 1.152 & $0.968-1.370$ & 0.111 & 1.215 & $0.626-2.358$ & 0.564 \\
\hline Preoperative oral carbohydrate & 0.917 & $0.751-1.121$ & 0.399 & 0.770 & $0.356-1.666$ & 0.507 \\
\hline Pre-admission counselling & 1.199 & $0.791-1.818$ & 0.391 & 1.781 & $0.314-10.124$ & 0.515 \\
\hline \multicolumn{7}{|l|}{ Surgical Approach } \\
\hline Open & Referent & - & - & Referent & - & - \\
\hline Laparoscopic & 0.667 & $0.581-0.767$ & $<0.001$ & 0.457 & $0.266-0.787$ & 0.005 \\
\hline Conversion to open & 0.797 & $0.609-1.043$ & 0.098 & 0.602 & $0.212-1.705$ & 0.339 \\
\hline Surgery for rectal cancer & 1.000 & $0.883-1.132$ & 0.996 & 0.807 & $0.498-1.309$ & 0.385 \\
\hline Duration of procedure (minutes) & 1.003 & $1.002-1.004$ & $<0.001$ & 1.006 & $1.003-1.009$ & 0.001 \\
\hline Total intravenous general anaesthesia & 0.941 & $0.840-1.053$ & 0.288 & 0.937 & $0.606-1.449$ & 0.769 \\
\hline Antibiotic prophylaxis & 0.598 & $0.383-0.933$ & 0.023 & 0.855 & $0.150-4.867$ & 0.859 \\
\hline $\begin{array}{l}\text { Compliance with ERAS guideline for } \\
\text { normothermia intraoperatively }\end{array}$ & 0.820 & $0.544-1.236$ & 0.342 & 1.273 & $0.244-6.630$ & 0.774 \\
\hline Developed hypothermia $\left(<36^{0}\right.$ Celsius $)$ & 1.199 & $1.044-1.378$ & 0.011 & 0.869 & $0.504-1.497$ & 0.613 \\
\hline $\begin{array}{l}\text { Compliance with avoidance of long-acting } \\
\text { opioids intraoperatively }\end{array}$ & 0.904 & $0.806-1.015$ & 0.086 & 0.721 & $0.461-1.128$ & 0.152 \\
\hline $\begin{array}{l}\text { Compliance with ERAS guideline for IV fluid } \\
\text { Day } 0\end{array}$ & 1.102 & $0.953-1.275$ & 0.189 & 1.553 & $0.880-2.738$ & 0.129 \\
\hline $\begin{array}{l}\text { Long-acting opioids given } 1 \text { st } 48 \text { hours } \\
\text { postoperatively }\end{array}$ & 1.034 & $0.843-1.267$ & 0.751 & 1.212 & $0.554-2.652$ & 0.630 \\
\hline Anastomotic leak & 1.851 & $1.488-2.304$ & $<0.001$ & N/A & N/A & N/A \\
\hline
\end{tabular}

Table IV: Proportion of complications and Clavien-Dindo classification

\begin{tabular}{lcccccccc}
\hline Complications & $\begin{array}{c}\text { Complication: } \\
\text { number and rate }\end{array}$ & \multicolumn{2}{c}{ Complication events as per Clavien-Dindo classification: number and rate } \\
\hline & & I & II & IIIa & IIIb & IVa & IVb & V \\
\hline Wound infection & $54(11.8)$ & $34(9.8)$ & $15(4.3)$ & $3(0.9)$ & $7(2.0)$ & $0(0)$ & $2(0.6)$ & $0(0)$ \\
Urinary tract infection & $17(3.7)$ & $8(2.3)$ & $9(2.6)$ & $0(0)$ & $0(0)$ & $0(0)$ & $0(0)$ & $0(0)$ \\
Lobar atelectasis & $6(1.3)$ & $3(0.9)$ & $2(0.6)$ & $0(0)$ & $0(0)$ & $1(0.3)$ & $0(0)$ & $0(0)$ \\
Pneumonia & $4(0.9)$ & $0(0)$ & $2(0.6)$ & $0(0)$ & $0(0)$ & $1(0.3)$ & $1(0.3)$ & $0(0)$ \\
Anastomotic leaks & $30(8.5)$ & $0(0)$ & $2(1.1)$ & $2(1.1)$ & $23(6.1)$ & $1(0.3)$ & $2(0.6)$ & $1(0.3)$ \\
Deep wound dehiscence & $10(3.1)$ & $0(0)$ & $2(1.1)$ & $1(0.3)$ & $5(1.4)$ & $0(0)$ & $1(0.3)$ & $1(0.3)$ \\
Bowel obstruction & $19(5.4)$ & $0(0)$ & $3(1.1)$ & $1(0.3)$ & $13(3.8)$ & $1(0.3)$ & $0(0)$ & $1(0.3)$ \\
Paralytic ileus & $31(8.3)$ & $12(3.5)$ & $14(4.0)$ & $0(0)$ & $3(0.9)$ & $1(0.3)$ & $0(0)$ & $0(0)$ \\
Other & $32(7)$ & $62(17.9)$ & $44(12.7)$ & $12(3.5)$ & $27(7.8)$ & $11(3.2)$ & $11(3.2)$ & $2(0.6)$
\end{tabular}

There was no difference in complication rates between patients undergoing surgery for benign and malignant disease and between colon and rectal procedures.

\section{Discussion}

Our study of 457 patients undergoing elective colorectal surgery within an ERAS programme is the first reported from the African continent. Compliance with the ERAS guide- lines is critical to improved perioperative outcomes. ERAS centres in HICs with compliance levels greater than $70 \%$ consistently report lower LOS and complication rates. ${ }^{7,8,17}$ In our study, the overall pre-and intraoperative compliance (95\% and 85\% respectively) was high at the commencement of the ERAS programme and was maintained throughout the 5-year study period. Although postoperative compliance was initially low, it improved progressively over the study period 
to reach $74 \%$ in year 5 . The key areas for poor postoperative compliance were: inability to meet the mobilisation targets on Day 0 and 1, delay in commencement of a full ward diet and prolonged use of intravenous fluids. Further qualitative research is required to understand reasons for sub-optimal adherence to these elements of the guidelines. The high pre- and intraoperative compliance and the improved postoperative compliance rates could reflect the benefit of the PDSA cycle which includes weekly data review, regular team meetings and change management processes. ${ }^{15}$ In keeping with the published literature we found that as overall compliance improved, there was a reduction in LOS and complications.

The median LOS of 5 days achieved in this study is similar to that reported from ERAS centres in HIC settings. ${ }^{7,8}$ It is also $30-50 \%$ lower than the LOS reported for colorectal surgery from non-ERAS centres. ${ }^{2}$ Few studies have analysed the effect of compliance to the individual care elements on outcome. Our large sample size and high levels of compliance facilitated this analysis. On regression analysis, we found longer LOS was significantly associated with patient age, theatre time, postoperative hypothermia and the occurrence of an anastomotic leak. A shorter LOS was associated with appropriate antibiotic prophylaxis and laparoscopic surgery.

The duration of theatre time in minutes was independently associated with increased LOS and complications. For every 30 -minute increase in theatre time, the risk of complications increases by $12 \%$ and $\operatorname{LOS}$ by $8 \%$, irrespective of the type of procedure. This supports the findings of Hang in a systematic review and meta-analysis in $2018(n=66)$ that showed longer theatre time is associated with increased LOS and complications. ${ }^{18}$ Given the adverse consequences of prolonged LOS and complications, further investigation would be useful to evaluate interventions targeted to reduce theatre time. Strategies should be aimed at improving theatre efficiency without compromising patient safety. Adding an intraoperative surgical pause to the surgical checklist may be of value.

Similar to other studies, on regression analysis we found that laparoscopic surgery, despite longer operative time, was strongly associated with shorter LOS and fewer complications than open surgery. This could be related to a reduction in the metabolic stress response seen with laparoscopic surgery and requires further investigation. ${ }^{19}$

The overall complication rate of $44 \%$ in our study is in keeping with that reported by other ERAS studies. Just over a third (34\%) of our complications were Clavien-Dindo grade I. UTI, atelectasis, pneumonia and wound infection are common complications after elective colorectal surgery and add significantly to morbidity and costs. ${ }^{20}$ These complications were minimal in our study. This probably reflects the benefits of early mobilisation, early removal of urinary catheters and appropriate antibiotic prophylaxis. There was no difference in complication rates between patients undergoing colonic and rectal procedures. As the difference in morbidity between proximal and distal rectal procedures is significant, we plan on a sub-analysis in the future. An anastomotic leak is a devastating complication. Reported rates vary between $1.8-19.2 \%$ and it is associated with a mortality of $6-22 \%{ }^{21}$ The anastomotic leak and mortality rates in our study were $6.5 \%$ and $1.1 \%$ respectively, which compares favourably with published rates in both the
ERAS and non-ERAS setting. This supports the evidence that the ERAS programme is not associated with a high anastomotic leak or mortality rates. ${ }^{7,8,21}$ The mortality rate $(1.1 \%)$ in our study is low when compared to published mortality rates in both the ERAS and non-ERAS setting. This is an encouraging finding and requires further investigation.

Increasing age was not associated with an increase in complications as has been reported in non-ERAS settings. ${ }^{22}$ This suggests that if elderly patients are optimised perioperatively, as occurs with the ERAS programme, complications could be reduced. Optimal perioperative intravenous fluid management is a key component of the ERAS programme, and compliance to the fluid guidelines is independently associated with reduced complications. ${ }^{23} \mathrm{In}$ our study, although we had a high compliance to the D0 fluid guidelines (78\%), we did not find an association between fluid compliance and complications. This may be related to the fact that less than $2 \%$ of the non-compliant group received more than $5 \mathrm{~L}$ on $\mathrm{D} 0$, which suggests that a range of between $3 \mathrm{~L}$ and $5 \mathrm{~L}$ may be safe in the ERAS setting, but this needs further investigation.

The results achieved in this study are similar to that from published data from HICs. Our study was an audit of the ERAS programme that was implemented in the private health sector in Cape Town, SA. If the programme can be scaled up in the private sector and similar results can be achieved, it has the potential to make an important contribution to health care in SA. We believe that the ERAS programme has the potential to improve perioperative care in the public sector, but this requires further implementation research.

\section{Conclusion}

Our results show that high compliance to the ERAS guidelines is possible in the private sector in SA and that a significant reduction in LOS can be achieved without placing the patient at a higher risk of complications.

\section{Acknowledgements}

The authors would like to thank the participating ERAS nurse coordinators, surgeons, anaesthetists, physicians, nurses, physiotherapists, dieticians, hospital management and the staff at Matley and Partners.

\section{Conflict of interest}

Ravi Oodit is a consultant for PangeaMed. He declares that this had no influence on the submitted work. All the other authors declare that they have no conflict of interest.

\section{Funding source}

The funding source had no influence on the study design, data collection, analysis, manuscript preparation or publication. The study received a grant from Discovery Health.

\section{Ethical approval}

The study complies with the Declaration of Helsinki (2018). Ethics approval was obtained from the University of Cape Town (HREC 271/2020).

\section{ORCID}

R Oodit (iD https://orcid.org/0000-0002-7296-2941

DA Constant (iD https://orcid.org/0000-0002-7176-9963

F Maree (iD https://orcid.org/0000-0003-1618-5057 
I Lorrimer (iD https://orcid.org/0000-0003-1564-002X

EK Dalwai (iD https://orcid.org/0000-0002-7296-2941

J Moodley (iD https://orcid.org/0000-0002-9398-5202

\section{REFERENCES}

1. Tevis SE, Kennedy GD. Postoperative complications: looking forward to a safer future. Clin Colon Rectal Surg. 2016;29(3):246-52. https://doi.org/10.1055/s-0036-1584501.

2. Khuri SF, Henderson WG, DePalma RG, et al. Determinants of long-term survival after major surgery and the adverse effect of postoperative complications. Ann Surg. 2005;242(3):326343. https://doi.org/10.1097/01.sla.0000179621.33268.83.

3. Kehlet $\mathrm{H}$. The surgical stress response: should it be prevented? Can J Surg. 1991;34(6):565-7.

4. Ljungqvist $O$. Insulin resistance and outcomes in surgery. J Clin Endocrinol Metab. 2010;95(9):4217-9. https://doi. org/10.1210/jc.2010-1525.

5. Gustafsson UO, Scott MJ, Schwenk W, et al. Enhanced recovery after surgery society. Clin Nutr. 2012 Dec;31(6):783800. https://doi.org/10.1016/j.clnu.2012.08.013.

6. Varadhan KK, Neal KR, Dejong $\mathrm{CH}$, et al. The enhanced recovery after surgery (ERAS) pathway for patients undergoing major elective open colorectal surgery: a meta-analysis of randomised controlled trials. Clin Nutr. 2010;29(4):434-40. https://doi.org/10.1016/j.clnu.2010.01.004.

7. Greco M, Capretti G, Beretta L, et al Enhanced recovery program in colorectal surgery: a meta-analysis of randomised controlled trials. World J Surg. 2014;38(6):1531-41. https:// doi.org/10.1007/s00268-013-2416-8.

8. Lee L, Mata J, Ghitulescu GA, et al. Cost-effectiveness of enhanced recovery versus conventional perioperative management for colorectal surgery. Ann Surg. 2015;262(6):1026-33. https://doi.org/10.1097/SLA.00000000 00001019 .

9. Roulin D, Donadini A, Gander S, et al. Cost-effectiveness of the implementation of an enhanced recovery protocol for colorectal surgery. BJS. 2013;8:1108-14. https://doi. org/10.1002/bjs. 9184 .

10. Nguyen X, Anderson W, Tracy W, et al. An economic evaluation of the enhanced recovery after surgery (ERAS) multisite implementation programme for colorectal surgery in Alberta. Can J Surg. 2016;59(6):6716.

11. Biccard B, Madiba T, Kluyts H, et al. Perioperative outcomes in the African surgical outcomes study: a 7-day prospective observational cohort study. Lancet. 2018;391(10130):158998. https://doi.org/10.1016/S0140-6736(18)30001-1.

12. McQueen K, Oodit R, Derbew M, et al. Enhanced recovery after surgery for low-middle income countries. World J Surg. 2018;42:950-2. https://doi.org/10.1007/s00268-018-4481-5.

13. Oodit R, Ljungqvist O, Moodley J. Can an enhanced recovery after surgery programme improve colorectal cancer outcomes in South Africa. S Afr J Surg. 2018;56(1):8-11. https://doi. org/10.17159/2078-5151/2018/v56n1a2320.

14. Oodit R, McQueen K. ERAS in LMIC: enhanced recovery after surgery - a complete guide to optimising outcomes. Springer Links. 2020;623-30.

15. Taylor MJ, McNicholas C, Nicolay C, et al. Systematic review of the application of the plan-do-study-act method to improve quality in healthcare. BMJ Qual Saf. 2014;23(4):290-8. https://doi.org/10.1136/bmjqs-2013-001862.

16. Dindo D, Demartines N Clavien P. Classification of surgical complications: a new proposal with evaluation in a cohort of 6336 patients and results of a survey. Ann Surg 2014;240(2):205-13. https://doi.org/10.1097/01. sla.0000133083.54934.ae.

17. The ERAS Compliance Group. The impact of enhanced recovery protocol compliance on elective colorectal cancer resection: results from an international registry. Ann Surg. 2015;261(6):1153-9. https://doi.org/10.1097/ SLA.0000000000001029.

18. Hang C. Prolonged operative duration is associated with complications - a systematic review and meta-analysis. J Surg Res. 2018;229:134-44. https://doi.org/10.1016/j. jss.2018.03.022.

19. Buunen M, Gholghesaei M, Veldkamp R, et al. Stress response to laparoscopic surgery - a review. Surg Endosc. 2020;18(7):1022-8. https://doi.org/10.1007/s00464-003-916 9-7.

20. Lingsma HF, BottleA, Middleton S, et al. Evaluation of hospital outcomes: the relation between length-of-stay, readmission, and mortality in a large international administrative database. BMC Health Serv Res. 2018;18:116. https://doi.org/10.1186/ s12913-018-2916-1.

21. Daams F, Luyer M, Lange J. Colorectal anastomotic leakage: aspects of prevention, detection and treatment. World J Gastroenterol. 2013;19(15):2293-7. https://doi.org/10.3748/ wjg.v19.i15.2293.

22. Jafari MD, Jafari F, Halabi WJ, et al. Colorectal cancer resections in the aging US population - a trend toward decreasing rates and improved outcomes. JAMA Surg. 2014;149(6):55764. https://doi.org/10.1001/jamasurg.2013.4930.

23. Brandstrup B, Tønnesen $\mathrm{H}$, Beier-Holgersen $\mathrm{R}$, et al Effects of intravenous fluid restriction on postoperative complications - comparison of two perioperative fluid regimens - a randomised assessor-blinded multicentre trial. Ann Surg. 2003;238(5):641-8. https://doi.org/10.1097/01. sla.0000094387.50865.23. 